\title{
Evaluation of cardiac autonomic functions in Children with Thalassemia Trait, Iron Deficiency Anemia and Iron Deficiency
}

\author{
Mecnun Cetin ${ }^{1}$, Nichal Moumin ${ }^{2}$, Bertan Karaboga ${ }^{2}$, Senol Coskun ${ }^{1}$, Huseyin Gulen ${ }^{3}$ \\ ${ }^{1}$ Department of Pediatric Cardiology, Celal Bayar University, Manisa, Turkey \\ ${ }^{2}$ Department of Pediatrics, Celal Bayar University, Manisa, Turkey \\ ${ }^{3}$ Department of Pediatric Hematology, Celal Bayar University, Manisa, Turkey
}

\begin{abstract}
Distruption of cardiac autonomic functions during diseases associated with anemia were reported in multiple studies previously. Objective in this study is to investigate heart rate variability (HRV) for evaluation of cardiac autonomic functions in children patients with Thalassemia trait (TT), iron deficiency anemia (IDA) and iron deficiency (ID) without anemia.

Twenty-five patients with TT, 25 patients with IDA, 25 patients with ID, and 25 healthy controls were assessed with 24 hour ambulatory electrocardiography recordings. The following time-domain indices were calculated; standard deviation values of all normal sinus R-R intervals for 24 hours (SDNN), standard deviation levels of all mean normal sinus $\mathrm{R}-\mathrm{R}$ interspaces over each 5-minute strip in the 24-hour enrollments (SDANN), the square root of the mean of the sum of squares of differences between adjacent RR intervals (RMSSD), percentage of difference between adjacent normal R-R intervals that are greater than $50 \mathrm{~ms}$ computed for the overall 24-hour ECG recording (pNN50). Re-evaluation regarding HRV were done in children with IDA after 4 months of treatment.

Although reduction in HRV parameters was detected in IDA group compared to ID, TT, and control groups, it was statistically not significant. The pNN50 was significantly low only in the IDA group $(\mathrm{p}=0.042)$. When ID group and TT group were compared with control group, it was found that HRV parameters were not affected.

In IDA group, SDNN, SDANN, RMSSD and pNN50 index were significantly higher after trea tment $(\mathrm{p}=0.002, \mathrm{p}=0.005$, $\mathrm{p}=0.005, \mathrm{p}=0.010$, respectively).

Our results indicate that cardiac autonomic activity are affected in IDA and improvement occurs after treatment. HRV parameters were found to be especially associated with hemoglobin and hematocrit values.
\end{abstract}

Key Words: Heart rate variability, Iron deficiency anemia, Thalassemia trait

\section{Introduction}

Iron deficiency (ID) is at the most encountered nourishment deficiency in the world and $15-20 \%$ of the whole population has iron deficiency anemia (IDA). The most dramatic health consequence of severe anemia, which is well documented, is increased risk of child mortality. ID leads to the neuromotor and cognitive deterioration, developmental delay even in the absence of anemia (1-3). In thalassemia trait (thalassemia minor), which is another prevalent hypochromic microcytic anemia, blood values are similar to those in IDA. These patients are often wrongly diagnosed as IDA and treated for ID in vain. In thalassemia trait (T'T), unlike IDA, red cell distribution width (RDW), free erythrocyte protoporphyrin, ferritin, serum iron and total iron binding capacity (TIBC) is normal. The final diagnosis is established by determining the increased $\mathrm{HbA}_{2}$ in hemoglobin electrophoresis (4).
Heart rate variability (HRV) corresponds to the change in interval of heart beat and it is a physiological condition. HRV is accepted as one of the principal predictive marker of autonomic nervous function of the heart and it has been examined for the last twenty years. The HRV measurement which is a non-invasive and easy technique to apply has been extensively studied. The variation of HRV with regards to the time and frequency has been shown to be comprehensively related with the autonomic nervous function of the heart. It is a well-known and described data that HRV is related with the cardiovascular disorders and mortality (5-7).

IDA is usually related with elevated sympathetic system activity and reduced parasympathetic function. Decreased HRV has been previously showed in pediatric patients with IDA (8). However, the relationship between childhood IDA and HRV is not studied extensively. Beside this, from the fact that 
age-associated differences in autonomic nervous system between adult age group and pediatric population prevents the generalization of reliability of findings. Autonomic dysfunction was observed in patients with $\mathrm{B}_{12}$ deficiency which progresses with anemia, sickle cell anemia, and thalassemia major (9). As far as we know from literature, there are studies regarding evaluation of cardiac autonomic function in IDA patient. However, there is not enough data in literature related to cardiac autonomic functions in patients with TT, and ID in which hemoglobin ( $\mathrm{Hb}$ ) levels are normal while the ferritin level is low. In our study, HRV and cardiac autonomic function were evaluated in children with TT, IDA and ID.

\section{Materials and Methods}

This study was conducted in Celal Bayar University Hospital, Department of Pediatric Clinic. The study was confirmed by the local ethical committee (03.03.2009/0042).

A total of 100 cases aged 5-18 (mean age 11.92 \pm 3.45 years), 39 of which (39\%) male and 61 (61\%) of which female were added in the study. 25 of these patients were those who had applied to Pediatric Clinic and diagnosed with IDA after the tests and examinations; another 25 patients were the ones without ID; the other 25 patients were the ones monitored in Hematology Unit because of 'TT. Finally, the control group consisted of 25 healthy children who had applied to the Pediatric Unit but did not have any systemic diseases or medication history.

Complete blood count, reticulocytes, peripheral blood smear, ferritin, vitamin $\mathrm{B}_{12}$, folate, serum iron, TIBC and transferrin saturations (TS) were studied in all of the groups.

Anemia is described as the state in which the $\mathrm{Hb}$ value is $-2 \mathrm{SD}$ lower than the mean $\mathrm{Hb}$ value in the normal population of the same age and gender, as completely described by the WHO. Diagnosis of anemia of the study group was determined according to the table 1 (10). The children whose $\mathrm{Hb}$ was within the normal range, but the ferritin levels were below the normal levels were diagnosed with ID. In order to have differential diagnosis between the IDA and TT in patients with microcytic hypochromic anemia, hemoglobin electrophoresis was carried out on patients with normal or mildly low levels of $\mathrm{Hb}$ but with normal levels of RBC, serum iron and ferritin, and they were diagnosed with TT.

The children with vitamin $\mathrm{B}_{12}$ or folic acid deficiency, sickle cell anemia, systemic or chronic diseases were excluded from the study.

All the patients went through 24 hour-Holter ECG and their HRV was monitored. A 4-month oral iron treatment was started in the IDA group. The hemogram, iron, TIBC and ferritin levels were checked in the $4^{\text {th }}$ month of the treatment and the 24hour Holter ECG monitorization was repeated.

\section{HRV Analysis}

24-hour ambulatory electrocardiographic recordings were obtained using a CardioScan DMS 300 holter recorder (CardioScan, Beijing, China). All cases were recommended to maintain the normal routine of their daily life. All recordings were visually examined and manually over read to verify beat classification by the same experienced cardiologist. Abnormal beats and areas of artifact were automatically and manually identified and excluded from the analysis. The HRV was identified with the software of the same device. The HRV measurements in our study were as follows: Standard deviation values of all normal sinus R-R intervals for 24 hours (SDNN); standard deviation values of all mean normal sinus R-R intervals for each 5-minute segment in the 24-hour recordings (SDANN); root mean squares of successive differences between normal sinus R-R intervals (RMSSD); percentage of difference between adjacent normal R-R intervals that are greater than $50 \mathrm{~ms}$ computed for the entire 24-hour ECG recording (pNN50).

\section{Statistical Analysis}

SPSS (Statistical Package for the Social Sciences 14.0) program was used for statistical analysis. The data were presented in arithmetic mean \pm standard deviation. A single factor or one-way ANOVA test, Kruskal-Wallis test, chi-square test and MannWhitney test were used to compare the groups. The statistical significance limit was taken as $\mathrm{p}<0.05$ for all tests.

\section{Results}

A statistically significant difference was detected between the mean of ages of the study groups $(p=0.008)$. This difference was found to be arising from the age difference between the IDA and T'T groups $(p=0.010)$. There was no significant difference when IDA, ID and TT groups were compared ( $p=0.17 ; p=0.48 ; p=0.65$ respectively) (Table 2 ).

When hematologic and biochemical parameters were compared, IDA group had the lowest $\mathrm{Hb}$ value. There was a statistically significant difference among groups in terms of mean $\mathrm{Hb}$ value $(\mathrm{p}<0.001)$. Mean MCV value was significantly lower in the IDA and TT group compared to the control group $(\mathrm{p}<0.001)$. Mean RDW value was significantly higher in the IDA group compared to the other groups $(p<0.001)$. While serum Fe, ferritin and TS values were significantly lower in the IDA group compared to the other 
Table 1. Normal age and gender related changes of $\mathrm{Hb}$ and Hct values for children.

\begin{tabular}{lllllllll}
\hline & FEMALE & \multicolumn{5}{c}{ MALE } \\
\hline & Hb(g/L) & & Hct $(\%)$ & & Hb(g/L) & Hct $(\%)$ \\
Age(years) & mean & $-2 S D$ & mean & -2 SD & mean & $-2 S D$ & mean & $-2 S D$ \\
$5-7.99$ & 125 & 109 & 37.2 & 33.0 & 125 & 109 & 37.2 & 33.0 \\
$8-11.99$ & 128 & 110 & 38.4 & 34.0 & 128 & 110 & 38.4 & 34.0 \\
$12-14.99$ & 134 & 115 & 39.0 & 34.0 & 140 & 120 & 40.5 & 35.0 \\
$15-18$ & 135 & 117 & 39.5 & 34.0 & 148 & 123 & 43.0 & 37.0
\end{tabular}

Table 2. Demographic, hematological and biochemical values in the groups.

\begin{tabular}{|c|c|c|c|c|c|}
\hline & $\begin{array}{l}\mathrm{IDA}(\mathrm{n}=25) \\
\text { Mean } \pm \text { SD }\end{array}$ & $\begin{array}{c}\mathrm{ID}(\mathrm{n}=25) \\
\text { Mean } \pm \text { SD }\end{array}$ & $\begin{array}{c}\mathrm{TT}(\mathrm{n}=25) \\
\text { Mean } \pm \text { SD }\end{array}$ & $\begin{array}{c}\text { Control }(\mathrm{n}=25) \\
\text { Mean } \pm \mathrm{SD}\end{array}$ & $\mathrm{p}$ \\
\hline Age(years) & $13.29 \pm 2.96$ & $12.71 \pm 3.66$ & $10.29 \pm 2.32$ & $11.38 \pm 4.01$ & 0.008 \\
\hline Sex (male/female) & $\begin{array}{c}7(28 \%) / 18(72 \\
\%)\end{array}$ & $6(24 \%) / 19(76 \%)$ & $14(56 \%) / 11(44 \%)$ & $\begin{array}{c}12(48 \%) / 13(52 \\
\%)\end{array}$ & 0.057 \\
\hline Weight $(\mathrm{kg})$ & $46.32 \pm 10.8$ & $42.76 \pm 14.4$ & $40.07 \pm 13.1$ & $44.23 \pm 11.4$ & 0.197 \\
\hline $\mathrm{RBC}(\mathrm{mm} 3)$ & $4.38 \pm 0.58$ & $4.67 \pm 0.45$ & $5.70 \pm 0.53$ & $4.77 \pm 0.33$ & $<0.001$ \\
\hline $\mathrm{Hb}(\mathrm{g} / \mathrm{dL})$ & $8.9 \pm 1.87$ & $12.9 \pm 1.01$ & $10.9 \pm 0.78$ & $13.4 \pm 0.93$ & $<0.001$ \\
\hline $\operatorname{Hct}(\%)$ & $28.7 \pm 5.33$ & $38.2 \pm 3.45$ & $34.5 \pm 2.80$ & $40.1 \pm 2.92$ & $<0.001$ \\
\hline $\operatorname{MCV}(\mathrm{fl})$ & $65.6 \pm 8.05$ & $82.4 \pm 3.33$ & $60.9 \pm 3.08$ & $84.3 \pm 3.89$ & $<0.001$ \\
\hline $\mathrm{RDW}(\%)$ & $19.6 \pm 4.00$ & $13.4 \pm 0.42$ & $13.8 \pm 0.87$ & $13.2 \pm 0.48$ & $<0.001$ \\
\hline Reticulocyte(\%) & $1.35 \pm 0.52$ & $1.19 \pm 0.41$ & $1.24 \pm 0.49$ & $1.06 \pm 0.22$ & 0.147 \\
\hline $\operatorname{Iron}(\mu \mathrm{g} / \mathrm{dL})$ & $14.8 \pm 15.6$ & $66.5 \pm 20.6$ & $103.8 \pm 43.1$ & $87.5 \pm 26.4$ & $<0.001$ \\
\hline $\operatorname{TIBC}(\mu \mathrm{g} / \mathrm{dL})$ & $368.0 \pm 71.5$ & $265.5 \pm 54.3$ & $243.7 \pm 53.6$ & $247.5 \pm 64.3$ & $<0.001$ \\
\hline Ferritin(ng/mL) & $2.80 \pm 1.32$ & $6.70 \pm 2.15$ & $26.3 \pm 12.6$ & $23.2 \pm 10.4$ & $<0.001$ \\
\hline $\mathrm{TS}(\%)$ & $4.07 \pm 4.73$ & $25.5 \pm 9.71$ & $39.1 \pm 13.6$ & $37.2 \pm 15.2$ & $<0.001$ \\
\hline Vit B12(pg/mL) & $218.4 \pm 135.1$ & $240.2 \pm 92.9$ & $286.1 \pm 165.6$ & $231.9 \pm 86.5$ & 0.249 \\
\hline Folic & $6.90 \pm 3.94$ & $5.97 \pm 1.83$ & $8.35 \pm 4.01$ & $7.01 \pm 2.84$ & 0.090 \\
\hline
\end{tabular}

$\operatorname{Acid}(\mathrm{ng} / \mathrm{mL})$

RBC, red blood cells; Hb, hemoglobin; Hct, hematocrit; MCV, mean corpuscular volume; RDW, red cell distribution width; TIBC, total iron-binding capacity; TS, transferrin.

SD: Standard Deviation.

groups $(\mathrm{p}<0.001)$, TIBC was significantly higher $(\mathrm{p}<0.001)$ (Table 2).

\section{Comparison of HRV indexes between groups}

There was no significant difference between groups in terms of mean heart rate $(p=0.416)$. There no significant difference between groups in terms of the lowest and the highest heart rates, either.

HRV parameters were lowest in the IDA group. When all groups were compared, the pNN50 was found to be significantly low only in the IDA group $(p=0.042)$. No statistically significant difference was detected in the IDA and the control groups in regard to SDNN, SDANN, RMSSD values $(\mathrm{p}=0.93, \mathrm{p}=1.00$, $\mathrm{p}=0.25$, respectively) (Table 3 ).

There was no statistically significant difference between the TT group and the control group in terms of SDNN, SDANN, RMSSD, pNN50 values $(\mathrm{p}=0.90, \mathrm{p}=0.87, \mathrm{p}=0.99, \mathrm{p}=0.99$, respectively) (Table 
3). In the same way, there was no statistically significant difference between the ID group and the control group in terms of SDNN, SDANN, RMSSD, $\mathrm{pNN} 50$ values $(\mathrm{p}=0.95, \mathrm{p}=0.88, \mathrm{p}=0.94, \mathrm{p}=0.91$, respectively) (Table 3).

When pre-treatment and post-treatment hematologic and biochemical values were compared in the IDA group, a statistically significant difference was detected between pre-treatment and post-treatment values in terms of all parameters (Table 4).

In IDA group, SDNN, SDANN, RMSSD and pNN50 index were significantly higher after treatment $(\mathrm{p}=0.002, \mathrm{p}=0.005, \mathrm{p}=0.005, \mathrm{p}=0.010$, respectively). Post-treatment minimum and mean heart rate were significantly low $(\mathrm{p}=0.006, \mathrm{p}<0.001$, respectively) (Table 5, Fig.1).

In IDA group, there were significant correlations between the SDNN, RMSSD and pNN50 index and $\mathrm{Hb}$ and hematocrit (Hct). However, serun iron and ferritin values were not correlated with the SDNN, RMSSD and pNN50 index in the patients with IDA (Table 6).

Table 3. HRV values of the groups.

\begin{tabular}{cccccc}
\hline & $\begin{array}{c}\mathrm{IDA}(\mathrm{n}=25) \\
\text { Mean } \pm \mathrm{SD}\end{array}$ & $\begin{array}{c}\mathrm{ID}(\mathrm{n}=25) \\
\text { Mean } \pm \mathrm{SD}\end{array}$ & $\begin{array}{c}\mathrm{TT}(\mathrm{n}=25) \\
\text { Mean } \pm \mathrm{SD}\end{array}$ & $\begin{array}{c}\text { Control(n=25) } \\
\text { Mean } \pm \mathrm{SD}\end{array}$ & $\mathrm{p}$ \\
\hline Minimum HR(beats/min) & $55.0 \pm 7.80$ & $51.0 \pm 6.49$ & $52.4 \pm 4.82$ & $51.2 \pm 5.36$ & 0.088 \\
Maximum HR(beats/min) & $155.0 \pm 9.83$ & $150.5 \pm 28.77$ & $159.6 \pm 18.16$ & $151.8 \pm 18.16$ & 0.459 \\
Mean HR(beats/min) & $90.9 \pm 7.69$ & $86.6 \pm 9.37$ & $89.4 \pm 8.16$ & $88.9 \pm 10.99$ & 0.416 \\
SDNN(ms) & $119.5 \pm 23.49 *$ & $129.2 \pm 34.64$ & $130.6 \pm 26.87$ & $124.8 \pm 36.6$ & 0.578 \\
SDANN(ms) & $108.2 \pm 23.68^{\wedge}$ & $114.4 \pm 30.81$ & $114.6 \pm 25.26$ & $108.5 \pm 34.61$ & 0.771 \\
RMSSD(ms) & $37.0 \pm 11.49 \&$ & $47.9 \pm 14.48$ & $46.6 \pm 17.01$ & $45.4 \pm 19.09$ & 0.071 \\
pNN50(\%) & $14.3 \pm 810$ & $22.9 \pm 11.67$ & $21.3 \pm 12.01$ & $20.8 \pm 12.63$ & 0.042
\end{tabular}

${ }^{* \wedge}$ \& There was no statistically significant difference between the control group $(p=0.93, p=1.0, p=0.25$ respectively). HR, heart rate; SDNN, standard deviations of all mean R-R intervals; SDANN: standard deviation of the mean normal-tonormal RR intervals; RMSSD, root mean square of the successive difference; pNN50: RR intervals greater than 50 ms. SD: Standard Deviation

Table 4. Hematological and biochemical parameters before and after treatment in IDA group.

\begin{tabular}{|c|c|c|c|}
\hline $\begin{array}{c}\text { IDA group }(n=25) \\
\text { Mean } \pm \text { SD }\end{array}$ & $\begin{array}{l}\text { Pre-treatment } \\
\text { Mean } \pm \text { SD }\end{array}$ & Post-treatment & $\mathrm{p}$ \\
\hline $\mathrm{RBC}\left(\mathrm{mm}^{3}\right)$ & $4.38 \pm 0.58$ & $4.72 \pm 0.44$ & 0.005 \\
\hline $\mathrm{Hb}(\mathrm{g} / \mathrm{dL})$ & $8.93 \pm 1.87$ & $12.86 \pm 1.22$ & $<0.001$ \\
\hline $\operatorname{Hct}(\%)$ & $28.72 \pm 5.33$ & $38.56 \pm 3.79$ & $<0.001$ \\
\hline $\operatorname{MCV}(\mathrm{fl})$ & $65.66 \pm 8.05$ & $81.08 \pm 4.09$ & $<0.001$ \\
\hline RDW $(\%)$ & $19.62 \pm 4.00$ & $14.73 \pm 1.42$ & $<0.001$ \\
\hline Reticulocyte $(\%)$ & $1.35 \pm 0.52$ & $1.76 \pm 0.54$ & 0.023 \\
\hline $\operatorname{Iron}(\mu \mathrm{g} / \mathrm{dL})$ & $14.88 \pm 15.66$ & $78.48 \pm 44.52$ & $<0.001$ \\
\hline $\operatorname{TIBC}(\mu \mathrm{g} / \mathrm{dL})$ & $368.6 \pm 71.5$ & $270.2 \pm 58.0$ & $<0.001$ \\
\hline Ferritin(ng/mL) & $2.80 \pm 1.32$ & $15.70 \pm 9.44$ & $<0.001$ \\
\hline $\mathrm{TS}(\%)$ & $4.07 \pm 4.73$ & $34.11 \pm 44.31$ & 0.003 \\
\hline
\end{tabular}

RBC, red blood cells; Hb, hemoglobin; Hct, hematocrit; MCV, mean corpuscular volume; RDW, red cell distribution width; TIBC, total iron-binding capacity; TS, transferrin. SD: Standard Deviation. 
Table 5. HRV parameters before and after treatment in IDA group.

\begin{tabular}{cccc}
\hline IDA group $(\mathrm{n}=25)$ & $\begin{array}{c}\text { Pre-treatment } \\
\text { Mean } \pm \mathrm{SD}\end{array}$ & $\begin{array}{c}\text { Post-treatment } \\
\text { Mean } \pm \text { SD }\end{array}$ & $\mathrm{p}$ \\
\hline Minimum HR(beats/min) & $55.08 \pm 7.80$ & $49.32 \pm 6.63$ & 0.006 \\
Maximum HR(beats/min) & $155.04 \pm 19.83$ & $165.48 \pm 23.00$ & 0.068 \\
Mean HR(beats/min) & $90.9 \pm 7.69$ & $83.92 \pm 8.81$ & 0.001 \\
SDNN(ms) & $119.52 \pm 23.4$ & $144.56 \pm 41.27$ & 0.002 \\
SDANN(ms) & $108.28 \pm 23.68$ & $130.68 \pm 40.83$ & 0.005 \\
RMSSD(ms) & $37.08 \pm 11.49$ & $46.76 \pm 15.54$ & 0.005 \\
pNN50(\%) & $14.32 \pm 8.10$ & $20.84 \pm 11.43$ & 0.010
\end{tabular}

HR, heart rate; SDNN, standard deviations of all mean R-R intervals; SDANN, standard deviation of the mean normalto-normal RR intervals; RMSSD, root mean square of the successive difference; pNN50, RR intervals greater than 50 ms. SD: Standard Deviation.

Table 6. Correlation with HRV parameters of $\mathrm{Hb}$ and hematocrit values in IDA group

\begin{tabular}{ccccc}
\hline & \multicolumn{2}{c}{ Hemoglobin/Hematocrit } & \multicolumn{2}{c}{ Ferrum/Ferritin } \\
\hline & $\mathrm{p}$ & Correlation coefficient & $\mathrm{p}$ & Correlation coefficient \\
\hline SDNN(ms) & $0.057 / 0.073$ & $0.385 / 0.365$ & $0.077 / 0.808$ & $0.361 / 0.051$ \\
SDANN(ms) & $0.131 / 0.171$ & $0.311 / 0.283$ & $0.056 / 0.517$ & $0.386 / 0.136$ \\
RMSSD(ms) & $0.007 / 0.008$ & $0.522 / 0.515$ & $0.932 / 0.246$ & $0018 / 0.241$ \\
pNN50(\%) & $0.008 / 0.009$ & $0.515 / 0.514$ & $0.988 / 0.295$ & $0.003 / 0.218$ \\
Mean HR & $0.018 / 0.021$ & $0.470 / 0.458$ & $0.583 / 0.240$ & $0.115 / 0.244$
\end{tabular}

SDNN, standard deviations of all mean R-R intervals; SDANN, standard deviation of the mean normal-to-normal RR intervals; RMSSD, root mean square of the successive difference; pNN50, RR intervals greater than 50 ms; HR, heart rate.

\section{Discussion}

HRV is a non-invasive and reliable method which allows the assessment and evaluation of the cardiac autonomous activity. With the interpretation of timeand frequency-bound parameters, one can obtain information about sympathetic and parasympathetic tonus. Decrease in HRV is a sign of autonomic dysfunction. Decreased SDNN, SDANN indices is a sign of sympathetic tonus increase. Decrease in time domain parameters $\mathrm{pNN} 50$ and RMSSD point to the decrease in parasympathetic effect. These findings were shown to increase the mortality risk $(11,12)$.

It was shown in many studies that in the diseases such as coronary failure, diabetes mellitus, and acute myocardial infarction that cardiac autonomic functions were impaired and it led to the decrease in
HRV (13-15). Lately, it has been pointed out that decreased HRV can prove significance in diagnosing

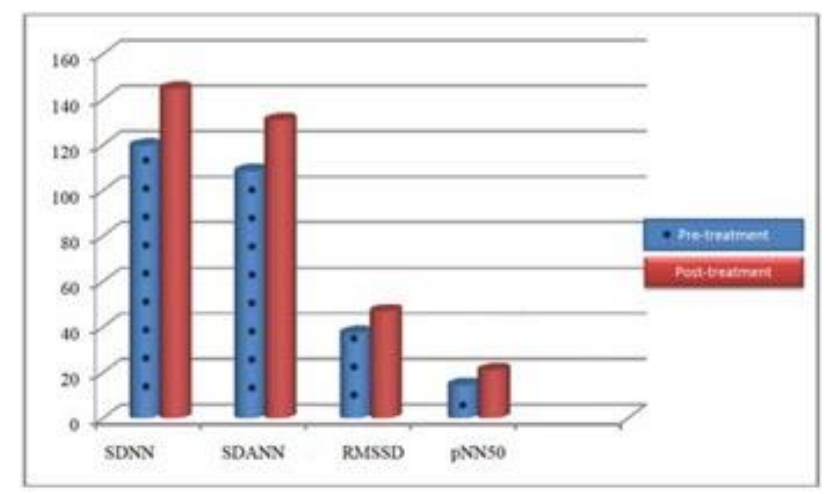

Fig. 1. Distribution of HRV parameters before and after treatment in IDA group. SDNN, standard deviations of all average R-R intervals; SDANN, standard deviation of the averaged normal-to-normal RR intervals; RMSSD, root mean square of the successive difference; pNN50, RR intervals greater than $50 \mathrm{~ms}$. 
sudden cardiac arrest and arrhythmia. Decrease in HRV was associated with the increase in mortality and morbidity in various cardiac diseases $(11,16,17)$.

The relationship between cardiac autonomic functions and anemia has been studied before in the anemic patient population (9). The correlation between anemia and HRV was researched in several anemia types such as thalassemia major, vitamin $B_{12}$ deficiency and sickle cell anemia. These studies suggested that the cardiac autonomic functions were impaired in patients with anemia (18-23). Yet, there are few studies related to secondary cardiac autonomic functions of ID, which is the main reason why anemia is prevalent in the society (9). Moreover, as far as we are concerned, there are no HRV studies in the literature related to patients with TT. There is increased evidence as to anemia leading to cardiac diseases and death in adults $(24,25)$. Decreased HRV in cardiac patients was independently shown to be associated with sudden cardiac death and mortality. Anemia was thought to contribute to the decrease in HRV in heart diseases (26). Yokuşoğlu et al. found that the time domain indices of HRV except RMSSD were lower in the working group compared to the control group in a study they conducted on 43 adult patients with IDA. It was considered that the decrease in HRV indices, especially in SDNN and SDANN, might be related to the increased sympathetic and parasympathetic activity (9). In our study, heart rate (HR) and HRV parameters indicative of cardiac autonomic system in T'T, IDA, and ID patients were compared to the control group. Additionally, the IDA findings were re-evaluated after oral iron treatment. In the IDA group, post-treatment mean HR and minimum HR was higher compared to the other groups, but there was no statistically significant difference. The time-domain pNN50 values of HRV were lower in the IDA group compared to the other groups (Table 3).

In the tests conducted with thalassemia major patients, all the time and frequency domain HRV parameters proved out to be significantly decreased in thalassemia major patients compared to the control group. These findings in the patients were considered to be potentially helpful in the preclinical stage of the heart disease where the sympathetic activity can be shown and in determining cardiac complications. Decreased HRV in chronic anemia-characterized patients with thalassemia major was due to sympathovagal activity disorder $(20,27,28)$. It was indicated that there could be decreases in early stages of the time and frequency domain parameters of HRV, even without clinical findings related to the cardiac functions of these patients $(18,19,29,30)$.

Although there are many HRV studies in the literature, conducted with thalassemia major patients, there are not any studies related to TT patients. In this study, HRV parameters of 25 TT patients who were not associated with ID were evaluated. There was no significant difference between the HRV time domain parameters and HR compared to the control group (Table 3). This finding makes us think that the TT autonomous nervous system is not affected, contrary to the patients with thalassemia major. We can think that TT does not pose an additional risk in terms of cardiac autonomic disorder.

In our study, when the ID group and the IDA group, whose $\mathrm{Hb}$ and Hct levels were normal but the body iron decreased, were compared, it was seen that the mean HR was higher in the IDA group while the HRV parameters were lower. This shows us that the $\mathrm{Hb}$ and Hct values might be more important than body-stored iron in terms of cardiac autonomic dysfunction. As we know of, there are no studies related to the patients with ID and without anemia. When the ID group was compared to the control group, it was seen that there was no change in the HRV parameters or mean HR because there was no difference in $\mathrm{Hb}$ and Hct values between the two groups (Table 3).

In a study conducted on 16 patients with renal failure and anemia, an HRV analysis was carried out in the initial stage and after treatment when the $\mathrm{Hb}$ values got back to normal. When the time domain analysis done on patients before treatment was compared with the control group, a significant decrease was detected (31).

In our study, the IDA group's hematologic, biochemical and HRV parameters before the treatment were compared with the ones after a 4month oral iron deficiency treatment. There were statistically significant differences in the pre- and post-treatment hematologic, biochemical and HRV parameters of the patients. The hematologic parameters of the patients, such as $\mathrm{Hb}$, Hct, and MCV and biochemical parameters such as iron and ferritin were statistically significantly higher compared to the pre-treatment values (Table 4). The mean HR and minimum HR was statistically significantly lower after treatment. Time domain HRV parameters such as SDANN, SDNN, RMSSD, pNN50 were statistically significantly lower before treatment compared to post-treatment (Table 5). In compliance with the limited number of previous studies, this means that cardiac autonomic functions are affected in IDA. It was shown that even in patients with no underlying chronic diseases, anemia alone caused decrease in HRV. While post-iron treatment there was significant recovery in $\mathrm{Hb}$ parameters of patients with IDA, there was recovery in HRV parameters at the same time. This shows that there is improvement in sympathovagal balance. A significant correlation between all parameters of HRV and Hb, Hct levels 
was detected (Table 6). All these findings suggest that ID treatment increases $\mathrm{Hb}$ and heals anemia while it increases cardiac parasympathetic activity. In patients with IDA, it is considered that the recovery from anemia also improves the sympathovagal balance and cardiac autonomic disorders can be prevented.

It is maintained in the literature that sympathetic activity is more dominant in IDA. This leads to the assumption that there might be deaths due to cardiovascular disease and arrhythmia-related deaths in this patient group. This hypothesis needs to be researched with the morbidity and mortality studies in the future (32). In our study, it was shown that the autonomic sympathetic and parasympathetic nerve activity was affected in patients with IDA and that the patients recovered from it after ID treatment. It was indicated that the HRV parameters were correlated with IDA, and a decrease in the HRV parameters of the patients with IDA was detected.

When evaluated with previous studies, it can be inferred from the data that the elevation in the need for oxygen in the tissue can result in elevation in sympathetic function and reduce in parasympathetic function. However, there are still not enough studies on children with IDA.

HRV analysis is considered to be a reliable technique in order to evaluate the autonomic activity in patients with cardiovascular disease. HRV's clinical use is limited except early diagnosis for diabetic autonomic neuropathy and post-myocardial infarction risk determination in patients. Despite all these researches, the physiological mechanism of HRV continues to be a debated issue. That is why the changes in HRV cannot be predicted most of the time and there is need for individual research in various clinical situations.

In light of all these findings and the results we obtained from our study, we can assert that IDA can lead to various autonomic nervous system disorders. In this study, the reason why there was a difference, but not of significant nature, between the compared IDA and control groups might be explained with the fact that the sample size was small, for as much as all the HRV parameters in the IDA group were low. In addition, there were statistically significant differences in pre- and post-treatment HRV parameters.

In conclusion, it was seen in our study that the mean HR and sympathetic activity increased in patients with IDA, while the parasympathetic activity was decreased and sympathovagal balance was lost. When the fact that there are not many studies on children especially with IDA, and there are even no studies on patients with TT in the literature is considered, it becomes obvious that this study provides important information in this matter.
ID and IDA, prevalent in every age group have serious effects on all organs and systems. This study showed that even the healthy-looking children with ID can be subclinically cardiac-affected, and the importance of early diagnosis and treatment was emphasized. What is more, it is obvious that more studies need to be carried out in this matter on IDA patients, especially with children.

\section{References}

1. Yoon SH, Kim DS, Yu ST, Shin SR, Choi du Y. The usefulness of soluble transferrin receptor in the diagnosis and treatment to iron deficiency anemia in children. Korean J Pediatr 2015; 58: 1519.

2. Black RE, Allen LH, Bhutta ZA, et al. Maternal and Child Undernutrition Study Group. Maternal and child undernutrition: global and regional exposures and health consequences. Lancet 2008; 371: 243-260.

3. Clark SF. Iron deficiency anemia: diagnosis and management. Curr Opin Gastroenterol 2009; 25: 122-128.

4. Urrechaga E. Red blood cell microcytosis and hypochromia in the differential diagnosis of iron deficiency and beta-thalassaemia trait. Int $\mathrm{J}$ Lab Hematol 2009; 31: 528-534.

5. Thayer JF, Yamamoto SS, Brosschot JF. The relationship of autonomic imbalance, heart rate variability and cardiovascular disease risk factors. Int J Cardiol 2010; 141: 122-131.

6. Chattipakorn N, Incharoen $\mathrm{T}$, Kanlop N, Chattipakorn S. Heart rate variability in myocardial infarction and heart failure. Int $\mathrm{J}$ Cardiol 2007; 120: 289-296.

7. Karason K, Mølgaard H, Wikstrand J, Sjöström L. Heart rate variability in obesity and the effect of weight loss. Am J Cardiol 1999; 83: 1242-1247.

8. Walter T. Effect of iron-deficiency anemia on cognitive skills and neuromaturation in infancy and childhood. Food Nutr Bull 2003; 24: 104-110.

9. Yokusoglu M, Nevruz O, Baysan O, et al. The altered autonomic nervous system activity in iron deficiency anemia. Tohoku J Exp Med 2007; 212: 397-402.

10. Centers for Disease Control (CCD). CDC Criteria for anemia in children and childbearing age women. MMWR Morb Mortal Wkly Rep 1989; 38: 400-404.

11. Heart rate variability: standards of measurement, physiological interpretation and clinical use. Task Force of the European Society of Cardiology and the North American Society of Pacing and Electrophysiology. Circulation 1996; 93: 10431065.

12. La Rovere MT, Bigger JT Jr, Marcus FI, Mortara A, Schwartz PJ. Baroreflex sensitivity and heartrate variability in prediction of total cardiac 
mortality after myocardial infarction. ATRAMI (Autonomic Tone and Reflexes After Myocardial Infarction) Investigators. Lancet 1998; 351: 478484.

13. Brunner-La Rocca HP, Esler MD, Jennings GL, Kaye DM. Effect of cardiac sympathetic nervous activity on mode of death in congestive heart failure. Eur Heart J 2001; 22: 1136-1143.

14. Surwit RS, Feinglos MN. Stress and autonomic nervous system in type II diabetes. A hypothesis. Diabetes Care 1988; 11: 83-85.

15. Casolo GC, Stroder P, Signorini C, et al. Heart rate variability during the acute phase of myocardial infarction. Circulation 1992; 85: 20732079.

16. Stein KM, Borer JS, Hochreiter C, et al. Prognostic value and physiological correlates of heart rate variability in chronic severe mitral regurgitation. Circulation 1993; 88: 127-135.

17. Anand IS, Kuskowski MA, Rector TS, et al. Anemia and change in hemoglobin over time related to mortality and morbidity in patients with chronic heart failure: results from Val-HeFT. Circulation 2005; 112: 1121-1127.

18. De Chiara B, Crivellaro W, Sara R, et al. Early detection of cardiac dysfunction in thalassemic patients by radionuclide angiography and heart rate variability analysis. Eur J Haematol 2005; 74 : 517-522.

19. Franzoni F, Galetta F, Di Muro C, et al. Heart rate variability and ventricular late potentials in beta-thalassemia major. Haematologica 2004; 89: 233-234.

20. Veglio F, Melchio R, Rabbia F, et al. Blood pressure and heart rate in young thalassemia major patients. Am J Hypertens 1998; 11: 539547.

21. Sözen AB, Demirel S, Akkaya V, et al. Autonomic dysfunction in vitamin B12 deficiency: a heart rate variability study. J Auton Nerv Syst 1998; 71: 2527.

22. Romero Mestre JC, Hernández A, Agramonte O, Hernández P. Cardiovascular autonomic dysfunction in sickle cell anemia: a possible risk factor for suddendeath? Clin Auton Res 1997; 7: 121-125.

23. Connes P, Martin C, Barthelemy JC, et al. Nocturnal autonomic nervous system activity impairment in sickle cell trait carriers. Clin Physiol Funct Imaging 2006; 26: 87-91.

24. Hegde N, Rich MW, Gayomali C. The cardiomyopathy of iron deficiency. Tex Heart Inst J 2006; 33: 340-344.

25. Petering DH, Stemmer KL, Lyman S, Krezoski S, Petering HG. Iron deficiency in growing male rats: a cause of development of cardiomyopathy. Ann Nutr Metab 1990; 34: 232-243.

26. Gehi A, Ix J, Shlipak M, Pipkin SS, Whooley MA. Relation of anemia to low heart rate variability in patients with coronary heart disease (from the Heart and Soul study). Am J Cardiol 2005; 95 : 1474-1477.

27. Gurses D, Ulger Z, Levent E, Aydinok Y, Ozyurek AR. Time domain heart rate variability analysis in patients with thalassaemia major. Acta Cardiol 2005; 60: 477-481.

28. Engle MA. Cardiacinvolvement in Cooley'sanemia. Ann N Y AcadSci 1964; 119: 694-702.

29. Kardelen F, Tezcan G, Akcurin G, Ertug H, Yesilipek A. Heart rate variability in patients with thalassemia major. Pediatr Cardiol 2008; 29: 935939.

30. Rutjanaprom W, Kanlop N, Charoenkwan P, et al. Heart rate variability in beta-thalassemia patients. Eur J Haematol 2009; 83: 483-489.

31. Furuland H, Linde T, Englund A, Wikström B. Heart rate variability is decreased in chronic kidney disease but may improve with hemoglobin normalization. J Nephrol 2008; 21: 45-52.

32. Vaishnav S, Stevenson R, Marchant B, et al. Relation between heart rate variability early after acute myocardial infarction and longtermmortality. Am J Cardiol 1994; 73: 653-657. 